\title{
Primary CNS Extranodal Marginal Zone B-Cell Lymphoma: A Case Series of 2 Patients Treated with External Beam Radiation Therapy
}

\author{
Rojine Ariani Leslie Ballas \\ Department of Radiation Oncology, Keck School of Medicine of University of Southern \\ California, Los Angeles, CA, USA
}

\section{Keywords}

B cell · Central nervous system lymphoma - Extranodal marginal zone lymphoma - Marginal zone B-cell lymphomas · Radiation therapy

\section{Abstract}

Primary CNS extranodal marginal zone B-cell lymphoma (MZBL) is a rare low-grade nonHodgkin lymphoma characterized predominantly by small B cells, plasma cells, monocytoid cells, and scattered large immunoblasts. As a slow-growing tumor that remains localized, primary CNS MZBL carries an excellent clinical prognosis. Here, we report two cases of primary CNS MZBL successfully treated using external beam radiation therapy along with a literature review.

\section{Introduction}

Marginal zone B-cell lymphomas (MZBL) account for approximately 7\% of all nonHodgkin lymphomas and are the third most common subtype after diffuse large B-cell lymphoma and follicular lymphoma [1]. Extranodal primary MZBL commonly arises from the mucosa-associated lymphoid tissue of various organs, with the stomach being the most frequently described site [2]. Rarely, extranodal MZBL may present as a primary lesion in nonmucosal-associated tissue. Involvement of the CNS by MZBL is an exceptionally rare example. Primary CNS extranodal MZBL carries an excellent prognosis when compared to other subtypes of primary CNS lymphoma, given that it exhibits an indolent course and remains localized for a long time [2]. While most other subtypes tend to be high-grade diffuse 


\section{Case Reports in Oncology}

\begin{tabular}{l|l}
\hline Case Rep Oncol 2021;14:725-732 \\
\hline DOI: 10.1159/000515780 & $\begin{array}{l}\text { @ 2021 The Author(s). Published by S. Karger AG, Basel } \\
\text { www.karger.com/cro }\end{array}$ \\
\hline
\end{tabular}

Ariani and Ballas: Primary CNS Extranodal Marginal Zone B-Cell Lymphoma

large B-cell lymphomas displaying aggressive behavior, MZBL is a low-grade lymphoma characterized principally by small B-cell lymphocytes admixed with plasma cells, monocytoid cells, and scattered large immunoblasts [3]. Here, we present 2 cases of primary CNS MZBL treated successfully with external beam radiation therapy (RT).

\section{Case Reports/Case Presentations}

\section{Case 1}

A 66-year-old female with an 8-year history of progressive ataxia and right-sided hearing loss with an initial MRI that was interpreted as a meningioma presented after she had worsening diplopia for over 2 years. In October 2017, neurological examination showed decreased hearing on the right side, diplopia, and impaired balance in tandem walking. An MRI revealed a dural-based extra-axial mass along the right tentorium extending into the cerebellopontine angle cistern, right cavernous sinus, and medial wall of the right middle cranial fossa (Fig. 1).

In January 2018, she underwent subtotal resection of what was believed to be a meningioma. Histopathologic examination demonstrated a diffuse proliferation of monomorphic small B cells. Immunohistochemistry findings were positive for CD20, PAX5, and Bcl-2; staining was insignificant for CD5, CD10, CD23, CD43, cyclin D1, Bcl-6, and CD103. PCR molecular analysis was positive for clonal IgH rearrangement and negative for clonal IgK rearrangement. These results were most representative of a low-grade small B-cell extranodal MZBL. Lumbar puncture yielded cytology consistent with the MZBL. Bone marrow biopsy consisted of $15 \%$ lymphoma cells. Staging PET-CT did not detect extracranial disease.

Following surgery, the patient completed 3 cycles of intrathecal cytarabine and 8 cycles of rituximab. The patient was dispositioned to radiotherapy. Gross tumor volume was identified and expanded by $3 \mathrm{~mm}$ to define a clinical target volume (CTV). Planning target volume
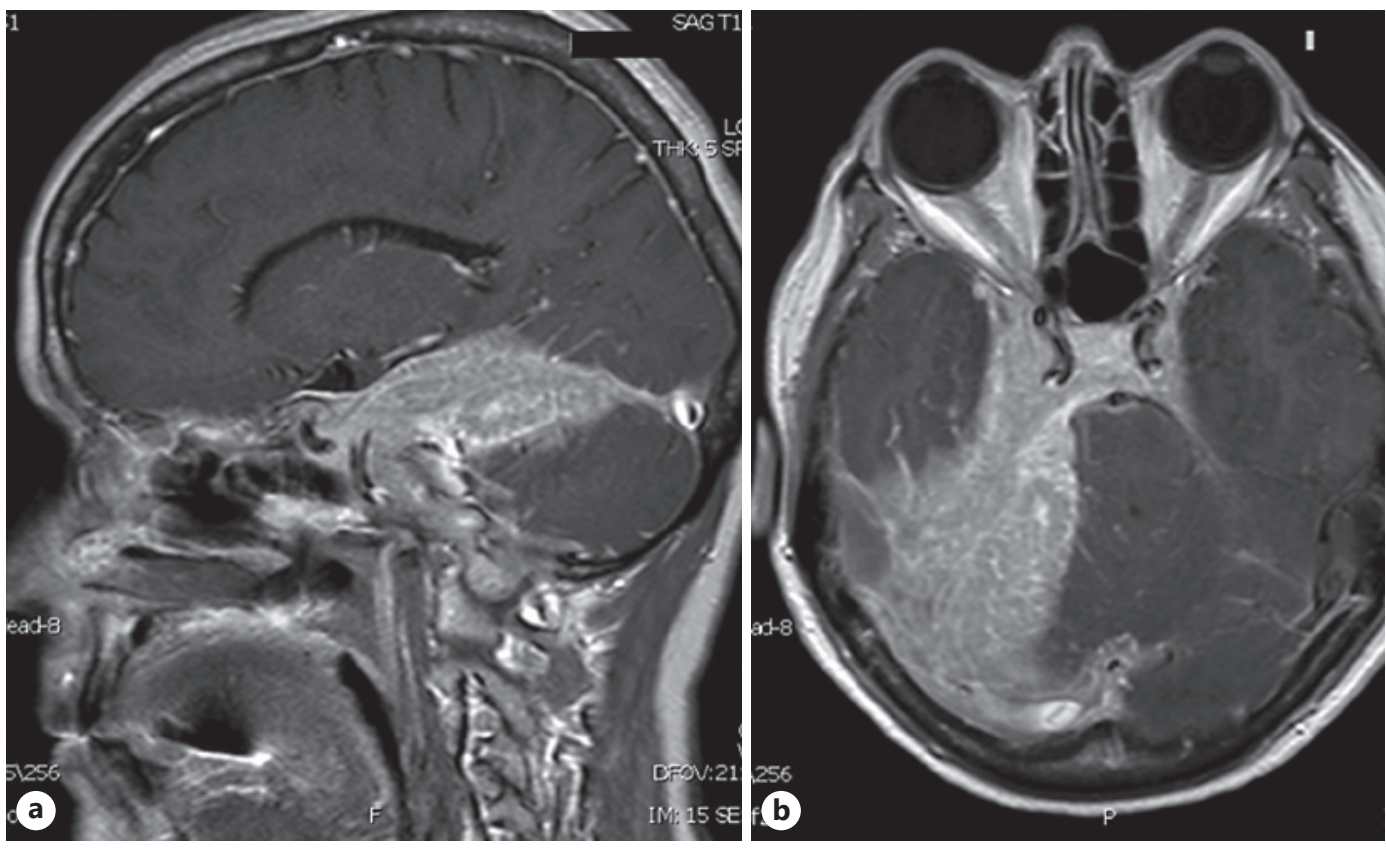

Fig. 1. Initial head MRI showing the enhancing extra-axial mass along the right tentorium in sagittal (a) and axial (b) planes. 


\section{Case Reports in Oncology}

\begin{tabular}{l|l}
\hline Case Rep Oncol 2021;14:725-732 \\
\hline DOI: 10.1159/000515780 & $\begin{array}{l}\text { @ 2021 The Author(s). Published by S. Karger AG, Basel } \\
\text { www.karger.com/cro }\end{array}$ \\
\hline
\end{tabular}

Ariani and Ballas: Primary CNS Extranodal Marginal Zone B-Cell Lymphoma

(PTV) was a 3-mm expansion on CTV to account for daily setup uncertainty. The patient received 24 Gy in 12 fractions to the PTV which she tolerated without any adverse effects.

Repeat MRI performed 1 month following completion of RT demonstrated a significant interval response to treatment with decrease in the size of the extra-axial mass and mass effect on the brainstem and posterior fossa (Fig. 2). No new areas of enhancement were detected. MRI performed 5 months following completion of RT demonstrated continued decrease in size of the extra-axial mass (Fig. 3). There were still no new areas of enhancement detected. At time of follow-up, the patient reported no new complaints and showed symptomatic improvement in her diplopia and gait instability, but no improvement in her hearing loss.

\section{Case 2}

A 55-year-old male patient with a history of chronic HBV infection and 1-month history of progressive right-sided weakness presented with acute-on-subacute weakness that began 1 week prior. Neurological examination demonstrated weakness of the right lower and upper extremities, headache, and sensations of dizziness and vertigo. MRI demonstrated multifocal peripherally enhancing cystic lesions involving the splenium and posterior body of the corpus
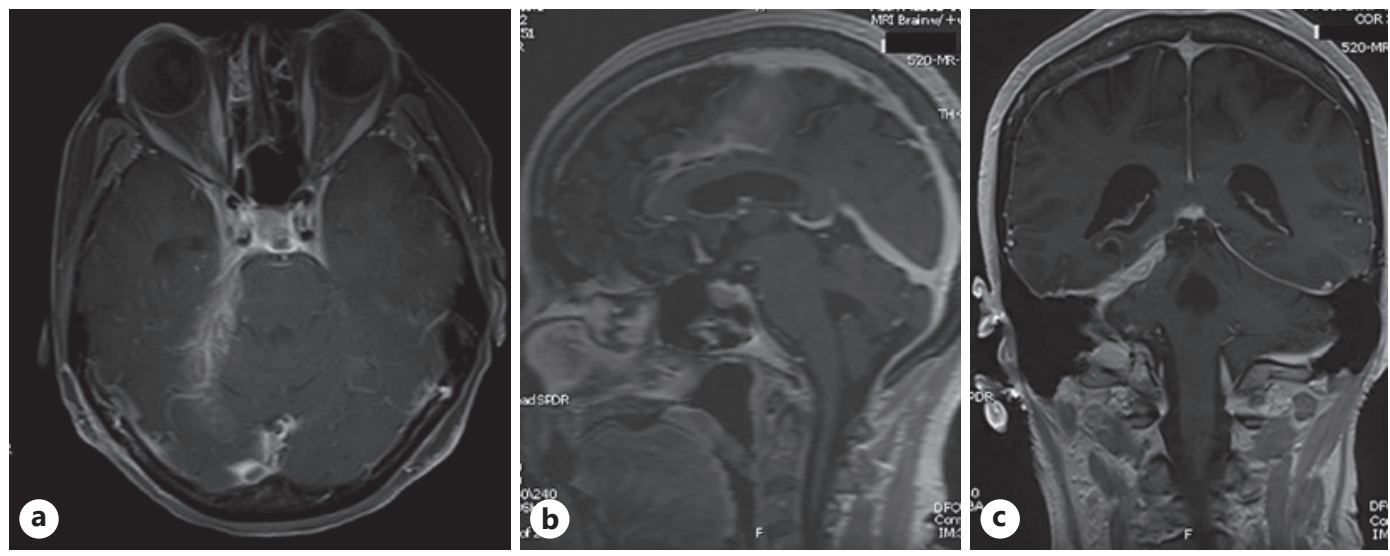

Fig. 2. MRI of the head 1 month following completion of RT showing decreased size of the extra-axial mass on the right with no new areas of enhancement in axial (a), sagittal (b), and coronal (c) planes. RT, radiation therapy.
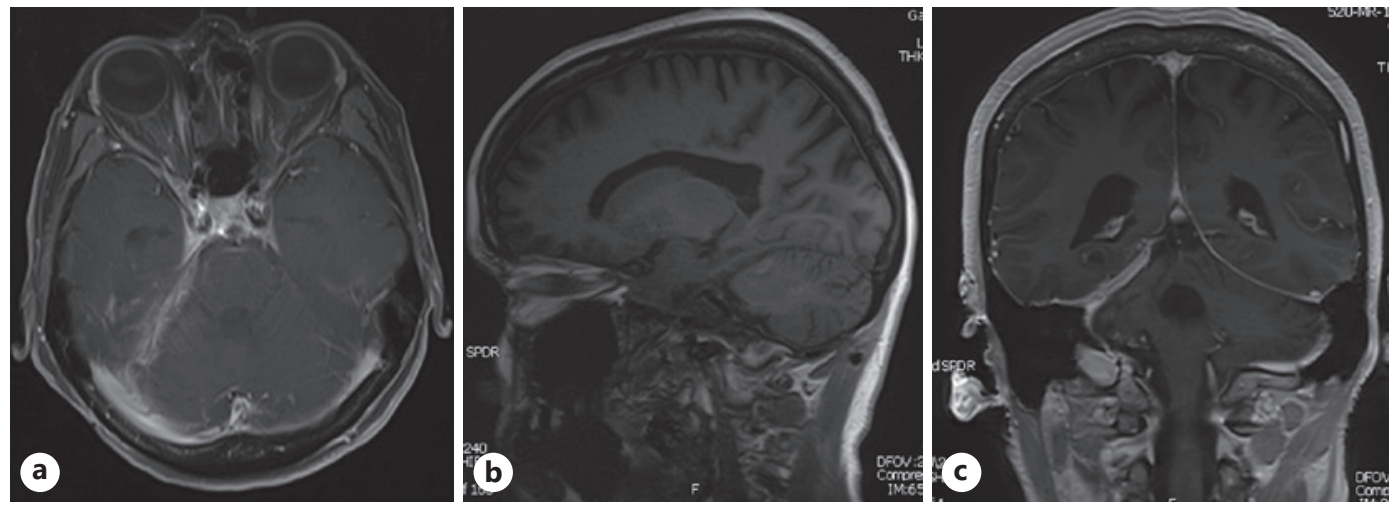

Fig. 3. MRI of the head 5 months following completion of RT showing further decrease in size of the extramass on the right, extending along the right tentorial leaflet with decreased leptomeningeal enhancement. No new areas of enhancement were detected in axial (a), sagittal (b), and coronal (c) planes. RT, radiation therapy. 


\section{Case Reports in Oncology}

\begin{tabular}{l|l}
\hline Case Rep Oncol 2021;14:725-732 \\
\hline DOI: 10.1159/000515780 & $\begin{array}{l}\text { @ 2 2021 The Author(s). Published by S. Karger AG, Basel } \\
\text { www.karger.com/cro }\end{array}$ \\
\hline
\end{tabular}

Ariani and Ballas: Primary CNS Extranodal Marginal Zone B-Cell Lymphoma

callosum, bilateral basal ganglia, and left corona radiata (Fig. 4). PET-CT scan demonstrated ill-defined lesions in the left posterior parietal lobe, right basal ganglia, and thalamus, with no evidence of extracranial disease. Differential diagnoses generated based on imaging findings included glioblastoma, lymphoma, and infection.

Lumbar puncture performed demonstrated large reactive-appearing immunoblasts and plasmacytoid lymphocytes, though no apparent malignant cells were identified. The patient subsequently underwent biopsy of the lesion, and immunohistochemical examination revealed positivity for $\mathrm{CD} 3, \mathrm{CD} 20, \mathrm{Bcl}-2$, and $k$ and $\lambda$ (polytypic pattern); staining was negative for CD5, CD10, CD23, CD43, cyclin D1, and Bcl-6. These findings were consistent with lowgrade extranodal CNS MZBL.

Following resection, the patient was treated with whole brain RT. CTV was defined to include all intracranial contents. PTV was a 7-mm expansion on the CTV to account for daily setup uncertainty. The patient received 24 Gy in 12 fractions to the PTV. During the course of treatment, he experienced dry eyes, nausea, and rash which were improved by artificial tears, ondansetron, and diphenhydramine and topical hydrocortisone, respectively. He also developed alopecia as a result of RT. Treatment was otherwise tolerated well without additional complications.
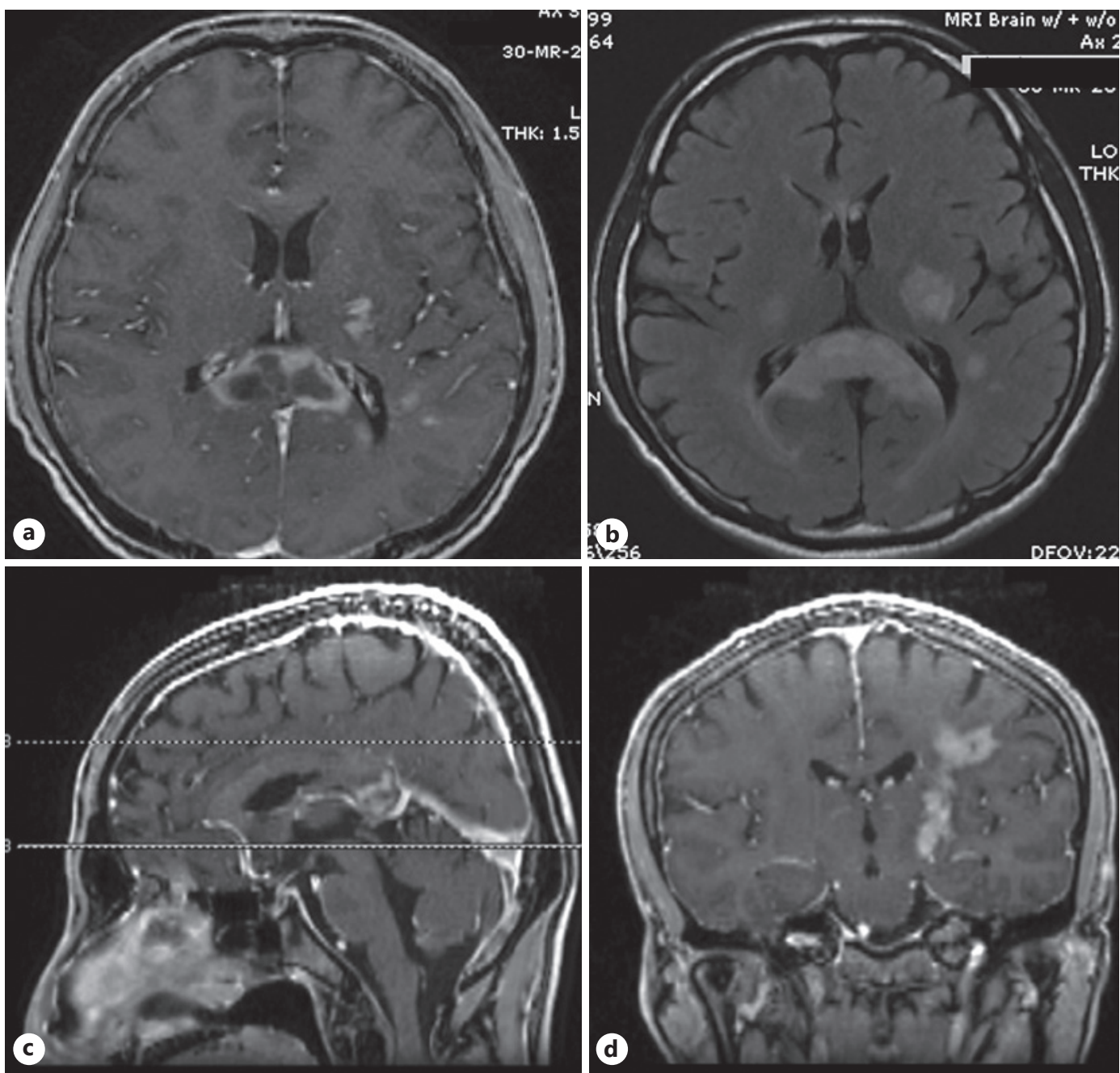

Fig. 4. Initial head MRI showing the multifocal peripherally enhancing lesions involving the splenium and posterior body of the corpus callosum, bilateral basal ganglia, and left corona radiata in axial (a, b), sagittal (c), and coronal (d) planes. 


\section{Case Reports in Oncology}

\begin{tabular}{l|l}
\hline Case Rep Oncol 2021;14:725-732 \\
\hline DOI: 10.1159/000515780 & $\begin{array}{l}\text { @ 2021 The Author(s). Published by S. Karger AG, Basel } \\
\text { www.karger.com/cro }\end{array}$ \\
\hline
\end{tabular}

Ariani and Ballas: Primary CNS Extranodal Marginal Zone B-Cell Lymphoma

Repeat MRI was performed 4 months following completion of RT and demonstrated significant interval response to treatment. There was comparatively marked decrease of hyperintensity involving the splenium of the corpus callosum and occipital horns and lateral margins of the lateral ventricle. Additionally, there was a notable decrease of hyperintensity in the left corona radiata extending into the left basal ganglia and left cerebral peduncle (Fig. 5). No new or residual areas of enhancement and no significant residual mass effect were detected. At time of follow-up, the patient reported no new complaints and demonstrated substantial symptomatic improvement of his right-sided weakness and dizziness with improved balance and walking. He also experienced improvement in his post-RT alopecia.

\section{Discussion/Conclusion}

The exact pathogenesis of primary CNS MZBL is poorly understood, as the CNS lacks mucosa-associated lymphoid tissue [4]. In certain non-CNS extranodal tissue sites, the development of MZBL has been described in association with specific chronic infectious
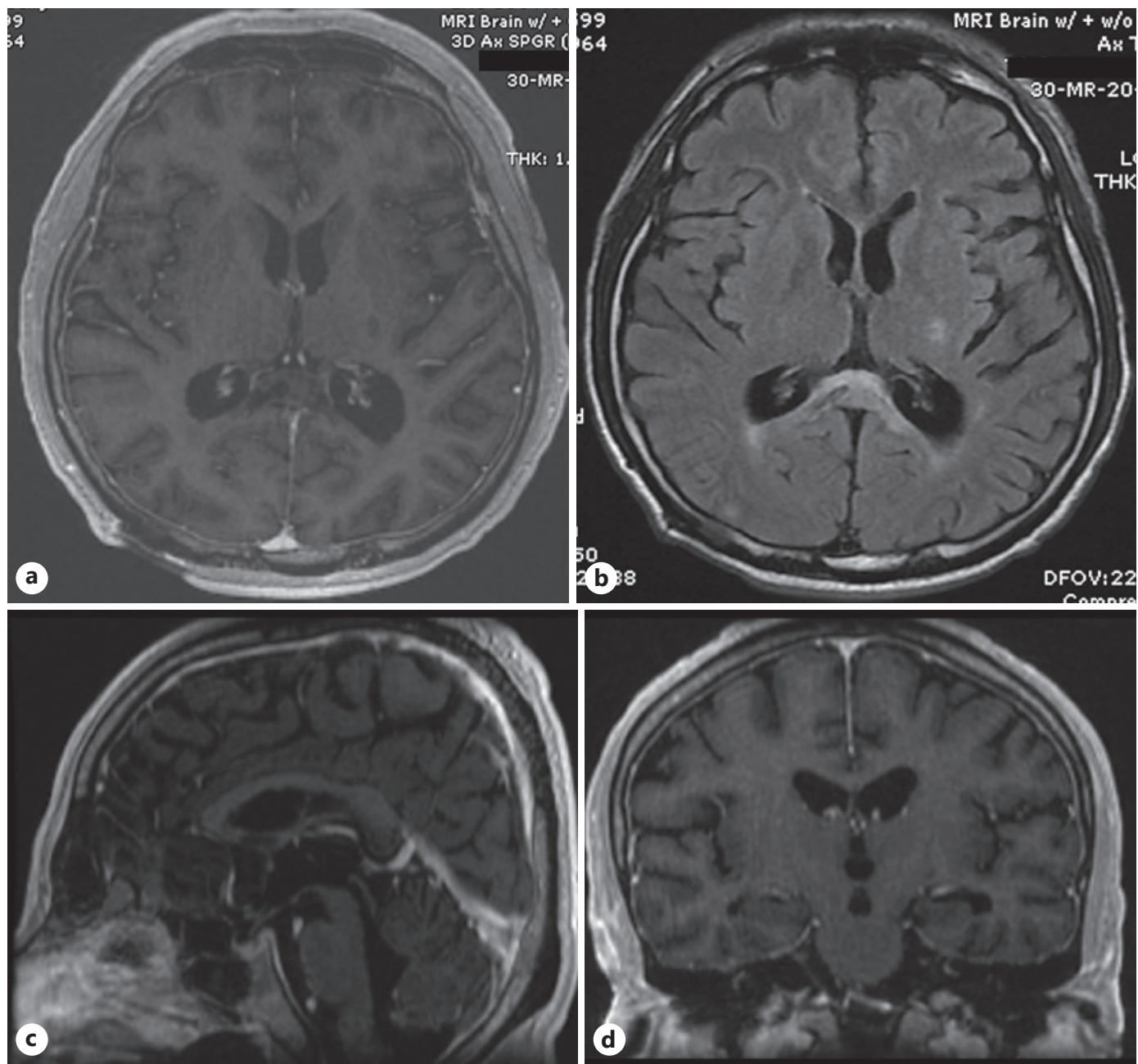

Fig. 5. MRI of the head 4 months following completion of CT showing decreased hyperintensity of the lesions involving the splenium and posterior body of the corpus callosum, bilateral basal ganglia, and left corona radiata in axial (a, b), sagittal (c), and coronal (d) planes. 


\section{Case Reports in Oncology}

\begin{tabular}{l|l}
\hline Case Rep Oncol 2021;14:725-732 \\
\hline DOI: 10.1159/000515780 & $\begin{array}{l}\text { ○ 2021 The Author(s). Published by S. Karger AG, Basel } \\
\text { www.karger.com/cro }\end{array}$ \\
\hline
\end{tabular}

Ariani and Ballas: Primary CNS Extranodal Marginal Zone B-Cell Lymphoma

and autoimmune processes, such as within gastrointestinal mucosa colonized by H.pylori and within lacrimal glands in Sjögren syndrome $[3,5]$. A small number of cases of patients with both HBV and MZBL have been reported in the literature, consistent with our second reported patient who presented with multifocal lesions and a long-standing history of untreated HBV [6]. This raises consideration for potential mechanisms behind the pathogenesis of CNS MZBL in our second patient, possibly arising either secondarily to direct chronic antigenic stimulation of the dura or as metastasis from an HBV-associated primary extracranial MZBL that went undetected on PET-CT. However, primary CNS MZBL has not been definitively proven to be directly linked to any infectious or autoimmune etiology and is in fact most frequently seen in immunocompetent middle-age females, consistent with the presentation of our first reported patient [4].

Detection and identification of primary CNS MZBL is of particular importance for prognosis and treatment planning. Due to the paucity of cases encountered, CNS MZBL is often initially misdiagnosed clinically and on imaging in nearly all cases, as occurred in both of our patients [7]. Primary CNS MZBL has most commonly been described in association with the dura matter [2]. Meningioma, the most frequently described primary CNS dural-associated lesion accounting for approximately $30 \%$ of all intracranial tumors, presents clinically and on MRI nearly identically to dural-based MZBL [5]. Both tumors commonly arise in cerebral convexities, appear isointense on T1-weighted MRI, and may demonstrate enhancing extraaxial lesions and dural tail sign [8]. T2-weighted imaging can be helpful in differentiating the two, as vasogenic edema can frequently be observed in tissue adjacent to dural-based MZBL $[8,9]$. Diffusion-weighted imaging is also useful in establishing a diagnosis, as MZBL demonstrates restricted diffusion due to its comparatively highly cellular nature [8]. Clinicians must be aware of these subtle differences in presentation when generating an initial differential based on imaging alone, as they play an essential role in successfully distinguishing primary CNS MZBL from more commonly encountered CNS tumors.

Nevertheless, imaging findings in primary CNS MZBL are nonspecific and cannot in isolation rule out alternate possible pathologies such as sarcoidosis, aspergillosis, or additional tumors including metastases, schwannomas, and chloromas [2]. Biopsy of the lesion is crucial in the definitive diagnosis of MZBL. As in our presented cases, tumors have been found to be composed of B cells positive for CD20 and clonal rearrangements of IgH and $k$, without expression of CD5, CD10, CD43, Bcl-6, and cyclin D1 [7, 10]. The presence of a low proliferation index in these cells is characteristic of the indolent nature of CNS MZBL.

Although there is currently no consensus on the optimal course of treatment of primary CNS MZBL due to the limited number of cases described in the available literature, the use of RT has been demonstrated to increase overall survival in affected patients [3, 10-15]. MZBL composed of CD20+ B cells is amenable to treatment with rituximab in conjunction with RT [10]. Additionally, the use of RT alone has also been shown to improve overall survival, without significant differences to outcomes of chemotherapy alone or combined chemo-RT [12]. The torpid and localized nature of primary CNS MZBL makes it an ideal candidate for external beam RT, necessitating only low doses to achieve significant clinical benefit [11]. In both of our patients, treatment with 24 Gy in 12 fractions to the PTV was tolerated without clinical complications and resulted in significantly decreased tumor size. Repeat posttreatment surveillance imaging performed on our first patient demonstrated no new areas of enhancement, highly suggestive of successful elimination of potential microscopic disease in adjacent tissue. This case presentation contributes to the small volume of existing literature supporting the utility of external beam RT in treatment of primary CNS MZBL.

We present 2 exceptionally rare cases of primary CNS MZBL initially misdiagnosed as more commonly encountered lesions presenting similarly on MRI imaging, emphasizing that MZBL should always be considered as a differential in context of imaging findings alone.

\section{Karger'}




\section{Case Reports in Oncology}

\begin{tabular}{l|l}
\hline Case Rep Oncol 2021;14:725-732 \\
\hline DOI: 10.1159/000515780 & $\begin{array}{l}\text { @ 2021 The Author(s). Published by S. Karger AG, Basel } \\
\text { www.karger.com/cro }\end{array}$ \\
\hline
\end{tabular}

Ariani and Ballas: Primary CNS Extranodal Marginal Zone B-Cell Lymphoma

Given that it is a particularly radiosensitive low-grade disease with an indolent clinical course, primary CNS MZBL carries an excellent prognosis and can be effectively treated with localized RT.

\section{Statement of Ethics}

All subjects included in this study have provided written informed consent to publish their cases and associated images.

\section{Conflict of Interest Statement}

The authors have no conflicts of interest to declare.

\section{Funding Sources}

This study received no financial support.

\section{Author Contributions}

Rojine Ariani, MS: conceptualization, writing - original draft, and writing - review and editing; Leslie Ballas, MD: conceptualization - review and editing, supervision, and project administration.

\section{References}

1 Zucca E, Arcaini L, Buske C, Johnson PW, Ponzoni M, Raderer M, et al. Marginal zone lymphomas: ESMO clinical practice guidelines for diagnosis, treatment and follow-up. Ann Oncol. 2020 01;31(1):17-29.

2 Sebastián C, Vela AC, Figueroa R, Marín MÁ, Alfaro J. Primary intracranial mucosa-associated lymphoid tissue lymphoma: a report of two cases and literature review. Neuroradiol J. 2014;27(4):425-30.

3 Goda JS, Gospodarowicz M, Pintilie M, Wells W, Hodgson DC, Sun A, et al. Long-term outcome in localized extranodal mucosa-associated lymphoid tissue lymphomas treated with radiotherapy. Cancer. 2010 Aug;116(16): 3815-24.

4 Bustoros M, Liechty B, Zagzag D, Liu C, Shepherd T, Gruber D, et al. A rare case of composite dural extranodal marginal zone lymphoma and chronic lymphocytic leukemia/small lymphocytic lymphoma. Front Neurol. 2018;9:267.

5 Pavlou G, Pal D, Bucur S, Chakrabarty A, van Hille PT. Intracranial non-Hodgkin's MALT lymphoma mimicking a large convexity meningioma. Acta Neurochir. 2006 Jul;148(7):791-3; discussion 93.

6 Ababou M, Mahtat EM, Jennane S, Elmaaroufi H, Mikdame M, Doghmi K. Splenic marginal zone lymphoma associated with hepatitis B virus infection, remission after viral treatment, and splenectomy: a case report and review of the literature. Hematol Oncol Stem Cell Ther. 2019 Jul 9.

7 Tu PH, Giannini C, Judkins AR, Schwalb JM, Burack R, O’Neill BP, et al. Clinicopathologic and genetic profile of intracranial marginal zone lymphoma: a primary low-grade CNS lymphoma that mimics meningioma. J Clin Oncol. 2005 Aug;23(24):5718-27.

8 Smith AB, Horkanyne-Szakaly I, Schroeder JW, Rushing EJ. From the radiologic pathology archives: mass lesions of the dura: beyond meningioma-radiologic-pathologic correlation. Radiographics. 2014 Mar-Apr; 34(2):295-312.

9 Elster AD, Challa VR, Gilbert TH, Richardson DN, Contento JC. Meningiomas: MR and histopathologic features. Radiology. 1989 Mar;170(3 Pt 1):857-62.

10 Lopetegui-Lia N, Delasos L, Asad SD, Kumar M, Harrison JS. Primary central nervous system marginal zone B-cell lymphoma arising from the dural meninges: a case report and review of literature. Clin Case Rep. 2020 Mar;8(3):491-7. 
11 Iwamoto FM, DeAngelis LM, Abrey LE. Primary dural lymphomas: a clinicopathologic study of treatment and outcome in eight patients. Neurology. 2006 Jun;66(11):1763-5.

12 Chihara D, Fowler NH, Oki Y, Fanale MA, Nastoupil LJ, Westin JR, et al. Impact of histologic subtypes and treatment modality among patients with primary central nervous system lymphoma: a SEER database analysis. Oncotarget. 2018 Jun; 9(48):28897-902.

13 de la Fuente MI, Haggiagi A, Moul A, Young RJ, Sidani C, Markoe A, et al. Marginal zone dural lymphoma: the Memorial Sloan Kettering Cancer Center and University of Miami experiences. Leuk Lymphoma. 2017 Apr; 58(4):882-8.

14 Sunderland AJ, Steiner RE, Al Zahrani M, Pinnix CC, Dabaja BS, Gunther JR, et al. An international multicenter retrospective analysis of patients with extranodal marginal zone lymphoma and histologically confirmed central nervous system and dural involvement. Cancer Med. 2020 Jan;9(2):663-70.

15 Karschnia P, Batchelor TT, Jordan JT, Shaw B, Winter SF, Barbiero FJ, et al. Primary dural lymphomas: clinical presentation, management, and outcome. Cancer. 2020 Jun;126(12):2811-20. 